\title{
Feasibility as a Gradual Notion
}

\author{
Libor Běhounek* \\ Institute of Computer Science \\ Academy of Sciences of the Czech Republic \\ Prague, Czech Republic \\ behounek@cs.cas.cz
}

\begin{abstract}
The notion of feasibility, applicable in various areas related to logic, is arguably of gradual nature - some tasks are more feasible than others. The possibility of modeling a gradual notion of feasibility in t-norm logics is brought forward and illustrated on the gradual notions of feasible number, feasible knowledge, feasible formula, and feasible computability.
\end{abstract}

\section{Introduction}

Feasibility is an important concept underlying many notions throughout logic as well as related disciplines such as computational complexity. It is modeled by various bivalent approximations: e.g., polynomial computability is often understood as a formal equivalent of computational feasibility. However, feasibility is clearly a gradual notion-some tasks are more feasible than others. None of the bivalent approximations captures the gradualness of feasibility, which limits their adequacy and usability. For example, polynomial computability with the degree of the polynomial larger than, say, $10^{100}$ can hardly be considered feasible. The bivalent division of tasks into feasible and unfeasible is thus a very crude one. In order to model feasibility more adequately, its gradual nature should be taken into account. The purpose of this note is to bring forward the possibility of modeling a gradual notion of feasibility by means of suitable non-classical logics.

\section{Logics of gradual notions}

Non-classical logics specifically designed for dealing with gradual notions are certain substructural logics known as t-norm logics (see esp. 4]). Gradual notions represented by their formulae are evaluated by degrees from the real unit interval $[0,1]$ (or other suitable algebras). Propositional connectives and quantifiers are in t-norm logics realized degree-functionally, i.e., by certain suitable operations on $[0,1]$. Tautologies are formulae always evaluated to the full degree 1.

Salient examples of t-norm logics are Łukasiewicz, Gödel, and product logic (denoted by $\mathrm{E}$, $\mathrm{G}$, and $\Pi$, respectively), which differ in the choice of operations for propositional connectives. In particular, conjunction is realized in $\mathrm{G}$ as the minimum of degrees, in $\Pi$ as their product, and in $\mathrm{E}$ as the operation $\min (0, x+y-1)$. Operations for other connectives are determined by that of conjunction in a certain natural way. Axiomatic systems sound w.r.t. [0,1]-valued semantics and complete w.r.t. a general algebraic semantics of these logics are known (for details see [4]).

\footnotetext{
* Supported by grant No. ICC/08/E018 of the Czech Science Foundation (part of the ESF EUROCORES program LogiCCC, project FP006 LoMoReVI) and grant No. IAA900090703 of the Grant Agency of the Academy of Sciences of the Czech Republic.
} 
There are also more general t-norm logics (e.g., BL and MTL) that cover all of the three above choices of connectives.

Implication in all t-norm logic evaluates to 1 iff the degree of its antecedent is lesser than or equal to the degree of its consequent. Consequently, tautologies of the form $A_{1} \& \ldots \& A_{n} \rightarrow B$ represent degree-preserving inference rules, transmitting the (lower estimates of) degrees from the \&-combination of the premises to the conclusion. First-order axiomatic theories can be developed over t-norm logics in the usual way (similarly as, e.g., over intuitionistic logic). Nonclassical mathematics based on t-norm logics (esp. a theory of gradual sets and relations - e.g., [3, 2]) has by now been developed to an extent sufficient for smooth application of the apparatus to gradual notions.

\section{Cost-based interpretation of t-norm logics}

T-norm logics can in principle serve for modeling any gradual notion. However, the fact that their connectives can in a natural way be interpreted in terms of costs or resources [1] makes them especially suitable for modeling the gradual notion of feasibility.

In the cost-based interpretation of t-norm logics, degrees represent costs of tasks denoted by formulae, with 1 representing no cost and 0 a maximal, infinite, or unaffordable cost (the ordering of costs is inverse to that of degrees - the lesser the degree, the larger the cost). Conjunction represents a combination of costs: Lukasiewicz conjunction corresponds to bounded addition of costs, product conjunction to unbounded addition (via logarithm), and Gödel conjunction to the maximum of costs (the latter is natural, e.g., for space complexity with erasable temporary memory). Other connectives also have natural meanings, derived from the meaning of conjunction: implication represents the remaining cost of the consequent, given the cost of the antecedent; negation the remainder (in terms of \&) to the maximal cost; etc. Costs of complex tasks are often expressible by suitable formulae of the appropriate t-norm logic. Tautologies of the form $A_{1} \& \ldots \& A_{n} \rightarrow B$ represent cost-preserving inference rules, transmitting the (upper estimates of) costs from the combination of the premise tasks to the conclusion task.

If the way how costs combine is known, the corresponding t-norm logic (usually, $\Pi, €$, or G) is to be used; otherwise a general logic covering all cases such as MTL or BL is applicable. If moreover the costs of all atomic tasks are known (e.g., the numbers of clock cycles of a processor per each instruction) and a mapping of costs to degrees is given, then we can directly calculate the degrees of complex tasks represented by formulae. The main advantage of applying the apparatus of t-norm logics, however, is the possibility of formulating axiomatic theories stating only general dependencies between task- and cost-combinations. Theorems of such theories over t-norm logics are applicable to all particular manners costs can be assigned (so, e.g., all particular processors), and can be developed in a way analogous to that of classical or intuitionistic mathematics. In particular, it is possible to design theories that capture a graded notion of feasibility, whose degrees are measured by the cost of performing the task (e.g., its time or space requirements): the larger the cost, the less feasible the task. This will be illustrated by four examples of graded notions of feasibility in areas related to logic-viz, feasible numbers, feasible knowledge, feasible formulae, and feasible computability.

\section{Feasible natural numbers}

A basic instance of gradual feasibility, to which all others can in principle be reduced, is that of feasible natural numbers. Natural axioms for the notion of feasible number (denoted by the 
predicate $\mathrm{FN}$ ) are the following:

$$
\begin{aligned}
& m<n \rightarrow(\mathrm{FN}(n) \rightarrow \mathrm{FN}(m)) \\
& \neg(\exists n) \neg \mathrm{FN}(n) \\
& \neg(\forall n) \mathrm{FN}(n)
\end{aligned}
$$

The axiom (11) expresses the natural fact that smaller numbers are more feasible than larger numbers, i.e., that the gradual set of feasible numbers is lower in the set of natural numbers (we understand feasibility as incremental accessibility from 0 , not in the sense that $10^{10^{100}}$ is more easily constructible than most $10^{100}$-digit numbers). The axiom (2) says that no natural number is completely unfeasible (see below for this reading of negation). This is justified by the fact that any standard natural number is incrementally accessible from 0 for a finite (though in some cases immensely large) cost, or the fact that for each $n$ there are infinitely many larger, so less feasible, numbers. The axiom (3) says that on the other hand, not all numbers are feasible: indeed, very large numbers (e.g., such that cannot even be expressed within the lifetime of the universe) can hardly be considered feasible.

Thus, all the three axioms (1)-(3) are quite natural requirements of the notion of feasible number. Observe, however, that the axioms (2) and (3) are contradictory in classical (i.e., bivalent, Boolean) logic. Classical logic thus cannot handle the notion of feasible number in this natural way. Nevertheless, the axioms (11)-(3) are consistent in t-norm logics with strict negation (i.e., negation interpretable as determinately not, yielding 0 for any non-zero degree, and 1 just for the degree 0) - e.g., in Gödel and product logic. The latter two logics are indeed appropriate for modeling, respectively, space-based and time-based feasibility (see Section 3). Even though there are no bivalent models of the axioms (11)-(3), gradual models in t-norm logics with strict negation do exist and show exactly the intuitively expected behavior, namely, that the degree of the feasibility of numbers decreases with increasing $n$, limiting to, but never reaching, the feasibility degree 0 .

The theory (11)-(3) does not assign particular degrees of feasibility to natural numbers (although they can be computed if particular resource-requirements and their mapping to degrees are fixed); general results on feasibility applicable to any particular manner of computation can be derived from these axioms as their consequences in MTL or another suitable t-norm logic.

\section{$5 \quad$ Feasible knowledge}

Standard accounts of epistemic logic endorse the principle of logical rationality, i.e., the assumption that the epistemic agent can perform elementary steps of logical deduction. In propositional epistemic logic, the principle is embodied in the axiom

$$
\mathrm{K} A \& \mathrm{~K}(A \rightarrow B) \rightarrow \mathrm{K} B
$$

for the epistemic modality $\mathrm{K} \varphi$ "the agent knows $\varphi$ ". However, this natural assumption about the agent's logical capability entails that the agent's knowledge is closed under the logical consequence relation, and consequently that the agent knows all theorems of propositional logic once its axioms are known to the agent. This unrealistic conclusion (consider, e.g., a complex tautology with a million variables) is called the logical omniscience paradox and is a major concern for epistemic logics.

For dealing with the logical omniscience paradox it is important to distinguish three types of the agent's knowledge: (i) actual knowledge actually available to the agent (e.g., the contents 
of its memory); (ii) potential knowledge that is in principle (disregarding resource limitations) derivable from the agent's actual knowledge; and (iii) feasible knowledge, which is realistically derivable from the actual knowledge. The logical omniscience paradox only regards feasible knowledge, since for actual knowledge the axiom (4) fails, and potential knowledge is indeed closed under logical consequence.

The paradox can be avoided by taking the gradualness of feasible knowledge into account. Degrees of the gradual modality $\mathrm{K}_{\mathrm{F}}$ then represent the cost of arriving at the knowledge (e.g., the time needed for its derivation). The axiom (44) then gets modified to the following form:

$$
\mathrm{K}_{\mathrm{F}} A \&\left(\mathrm{~K}_{\mathrm{F}} A \rightarrow \mathrm{K}_{\mathrm{F}} B\right) \& \mathrm{~F}_{\mathrm{MP}} \rightarrow \mathrm{K}_{\mathrm{F}} B
$$

where the propositional constant $\mathrm{F}_{\mathrm{MP}}$ represents the cost of performing a derivation by modus ponens. The use of an appropriate t-norm logic for the gradual modality $\mathrm{K}_{\mathrm{F}}$ (usually, $\mathrm{E}$ or $\Pi$, cf. Section 3) then eliminates the paradox, since the degree lapse given by $\mathrm{F}_{\mathrm{MP}}$ accumulates in longer derivations by the non-idempotent conjunction \&.

The gradual account of feasible knowledge also easily accommodates, e.g., the different feasibility of positive and negative introspection as well as other phenomena related to resourceaware reasoning about knowledge.

\section{Feasible formulae}

Classical metamathematics uses inductive definitions of formulae that do not take the feasibility of their forming into account. While this is quite legitimate in abstract mathematics, it becomes inadequate if the process of formula-forming is to be regarded-e.g., in knowledge representation of real-world agents, for whom extremely long formulae (e.g., of length $10^{10^{100}}$ ) are entirely unfeasible. Feasible formulae (FF) are definable as a gradual notion, with the inductive definition adjusted to reflect the cost of their forming similarly as in Section 5 ; e.g., $\mathrm{FF}(\varphi) \& \mathrm{FF}(\psi) \& \mathrm{~F}_{\mathrm{I}} \rightarrow \mathrm{FF}(\varphi \supset \psi)$. Metamathematical theorems (e.g., the substitution theorem, etc.) are then to be derived by means of a t-norm logic rather than classical logic. Preliminary results show that the basics of classical metamathematics can mostly be reproduced (with minor adjustments in rare cases) in MTL.

Developing metamathematics in t-norm logics opens the way for investigating various gradual metamathematical notions - e.g., the notion of (un)feasible (in)consistency of a theory (cf. the classical non-standard treatment of feasible numbers and feasible consistency in [5]).

\section{$7 \quad$ Feasible computability}

As stated in the Introduction, polynomial computability is often regarded as approximating the notion of feasible computability; however, the latter is clearly a gradual notion that is only roughly approximated by the former, since, e.g., computability in $O\left(n^{10^{100}}\right)$ time can hardly be considered effective.

A more realistic, gradual notion of feasibility of the polynomially computable problem can be based on the feasibility degrees assigned to the degree and coefficients of the polynomial (axiomatized by axioms similar to (11)-(3), rather than explicitly given). Then we have a gradual class of feasibly polynomial problems FP, theorems on which can be proved by means of t-norm logics. Other gradual classes of problems that take feasibility into account can be defined and studied by means of t-norm logics as well: e.g., the class NFP of non-deterministic feasibly polynomial problems, FPSPACE of problems solvable in feasibly polynomial space, etc. First- 
or higher-order t-norm logic again provides a ready-to-use apparatus for investigating these gradual notions of feasible computability.

Besides the formal apparatus, the gradual setting provides a different perspective on the complexity phenomena. For instance, the heuristic fact that mathematical results on space complexity are usually easier to obtain than those on time complexity can in the gradual view be informally explained by observing that space complexity uses (contractive) Gödel logic, which is much simpler than (non-contractive) product or Eukasiewicz logic associated with time complexity (cf. Section B).

\section{Conclusion}

In this short note I aimed to demonstrate that more realistic gradual notions of feasibility than those usually modeled in bivalent classical logic can be defined and studied by means of t-norm logics, which are specifically suitable for modeling gradual or cost-aware predicates and modalities. The price for the better adequacy is the use of non-classical (t-norm) logics for reasoning about the gradual notion. Nevertheless, reasoning in t-norm logics can be trained as easily as, e.g., intuitionistic reasoning about constructive notions. The apparatus of t-norm logics is sufficiently developed and available for a rather smooth mathematical work in t-norm logics (cf. esp. [3, 2]), so the development of a non-classical rather than classical theory of gradual feasibility comes at a rather low cost.

The study of gradual feasibility in the areas indicated above is a work in progress. The purpose of this short note was not to present particular technical results, but only to put forth a new approach to modeling feasibility as a gradual notion and point out some areas of its applicability. Preliminary results achieved so far show that this line of investigation is indeed viable and that most results on usual classical approximations of the notion of feasibility can be reproduced or refined by means of t-norm logics.

\section{References}

[1] L. Běhounek. Fuzzy logics interpreted as logics of resources. In M. Peliš, editor, The Logica Yearbook 2008, pages 9-21. College Publications, 2009.

[2] L. Běhounek, U. Bodenhofer, and P. Cintula. Relations in Fuzzy Class Theory: Initial steps. Fuzzy Sets and Systems, 159:1729-1772, 2008.

[3] L. Běhounek and P. Cintula. Fuzzy class theory. Fuzzy Sets and Systems, 154(1):34-55, 2005.

[4] P. Hájek. Metamathematics of Fuzzy Logic, volume 4 of Trends in Logic. Kluwer, Dordercht, 1998.

[5] R. Parikh. On existence and feasibility in arithmetic. Journal of Symbolic Logic, 36:494-508, 1971. 